\title{
CDISC SDTM Collected Race Terminology
}

National Cancer Institute

\section{Source}

National Cancer Institute. CDISC SDTM Collected Race Terminology. NCI Thesaurus.

Code C128689.

Terminology associated with the race collected codelist of the Clinical Data Interchange Standards Consortium (CDISC) Study Data Tabulation Model (SDT M). 